\title{
Back to the basics: The fundamentals of cryo-electron microscopy
}

Terje Dokland

University of Alabama at Birmingham, Birmingham, AL 35294

Cryo-electron microscopy (cryo-EM) was developed to allow the detailed structural analysis of complex biological matter in its native environment, with as few distortions and artifacts as possible. This tutorial will cover the fundamental principles and early development of cryo-EM, as well as practical aspects of cryo-EM sample preparation and imaging, including contrast formation and radiation damage.

All biological specimens are aqueous. Although technically possible, observation of fully hydrated samples in the EM is difficult due to the necessity for high vacuum inside the microscope column. Until the advent of cryo-EM, this meant that EM samples had to be completely dehydrated, typically leading to a number of artifacts associated with the removal of water, such as distortions, flattening, disruption of structural integrity and mass loss (Fig. 1A).

The technique of cryo-EM as currently performed was developed by Jacques Dubochet and co-workers at the European Molecular Biology Laboratory (EMBL) in the 1980s [1-3]. Dubochet had made two critical observations: firstly, that the vapor pressure of water at low temperature is so low that it remains stable under vacuum for prolonged periods of time. Secondly, he noted that water frozen very rapidly to below the "vitrification temperature" would not form ice crystals, but remained in a disordered, glass-like ("vitreous") state similar to liquid water. From this starting point, modern cryo-EM was born.

The most common method for sample vitrification is by plunging into liquid ethane at $<-150^{\circ} \mathrm{C}$ (liquid nitrogen is not suitable, due to the formation of an insulating gas layer that slows the cooling rate). Samples are transferred to the microscope and observed at liquid nitrogen temperatures using a special holder $[4,5]$. Cryo-EM thus allows the visualization of biological material in its native, hydrated state, without distortions and without the artifacts introduced by heavy atom staining (Fig. 1B). Cryo-EM also reveals the full three-dimensional distribution of density in the specimen, while traditional negative staining is dependent on the penetration of the stain into internal spaces in the specimen.

In combination with three-dimensional (3D) reconstruction and averaging techniques cryoEM has been used to solve protein and virus structures at near-atomic resolution [6]. At the other end of the scale, tomographic reconstruction has allowed the reconstruction of individually unique objects with no symmetry, such as pleiomorphic viruses (e.g. HIV), organelles like mitochondria, and entire cells, albeit at much lower resolution, providing a link between the molecular size range and that achievable by light microscopy $[7,8]$.

However, the cryo-EM technique is not without its problems and challenges. Since unstained, biological matter consists mostly of low atomic weight elements that distinguish themselves little from the background, frozen-hydrated samples display extremely low scattering contrast. By a suitable application of lens defocus, small mass-thickness dependent phase shifts are converted to observable amplitude differences. However, the defocus leads to phase inversions and a general deterioration in resolution that will need to be compensated computationally, if high resolution is to be attained. A field emission gun (FEG) microscope is essential for high resolution cryo-EM, as it allows the information limit to be extended, even at high defocus. Another big problem with biological specimens is their susceptibility to radiation damage, which means that radiation must be limited to only that required to form an detectable image. Fortunately, radiation 
sensitivity is reduced at the liquid nitrogen temperatures used in cryo-EM. The even lower temperature of liquid helium should in principle reduce the radiation damage even further; measurements have indicated that radiation damage is reduced by a useful but not overwhelmingly impressive factor of two.

An important innovation for the preparation of bulk samples was the introduction of highpressure freezing, developed in Basel in the 1990s [9, 10]. At high pressures, the vitrification temperature is increased, allowing thicker specimens like cells and tissues to be vitrified to a depth of several microns. Energy filtered EM has also been introduced and has proven to be particularly useful to image thick specimens where the increased amount of inelastic scattering is detrimental to the image quality.

Since its early development in the 1970s and 1980s, cryo-EM has matured to become the method of choice for the imaging of virtually all biological structures over an impressive range of sizes, ranging from individual protein molecules and complexes to cells and tissues.

1. Dubochet, J. and A.W. McDowall (1981). J. Microsc., 124, RP3-4.

2. $\quad$ Dubochet, J., et al. (1988). Quart. Rev. Biophys., 21, 129-228.

3. Lepault, J., F.P. Booy, and J. Dubochet (1983). J. Microscopy, 129, 89-102.

4. Dokland, T. and M.L. Ng, In Techniques in microscopy for biomedical applications., T. Dokland, et al., Editors. 2006, World Scientific Press: Singapore.

5. Grassucci, R.A., D.J. Taylor, and J. Frank (2007). Nat. Protoc., 2, 3239-3246.

6. Zhou, Z.H. (2008). Curr. Opin. Struct. Biol., 18, 218-228.

7. Lucic, V., F. Forster, and W. Baumeister (2005). Annu. Rev. Biochem., 74, 833-865.

8. McIntosh, R., D. Nicastro, and D. Mastronarde (2004). Trends Cell Biol., 15, 43-51.

9. $\quad$ Dubochet, J. (1995). Trends Cell Biol., 5, 366-368.

10. Vanhecke, D., W. Graber, and D. Studer (2008). Methods Cell Biol., 88, 151-164.
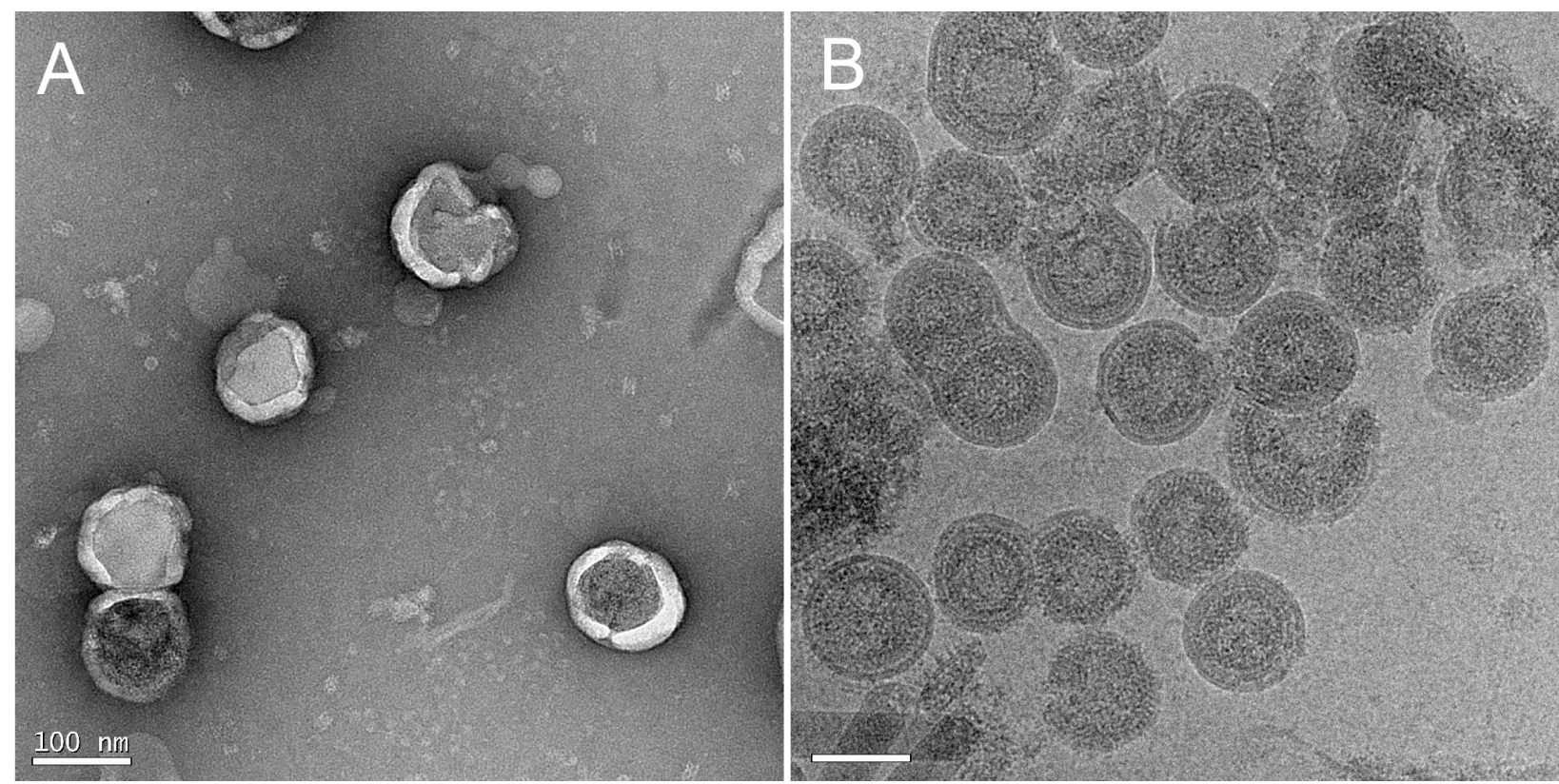

Fig. 1. HIV virus-like particles prepared by negative staining with uranyl acetate (A) and by cryoEM (B). The negatively stained sample displays considerable distortion of the shape and flatteting onto the grid and reveals few internal features, except in disrupted particles. By cryo-EM, on the other hand, sample shape is perfectly preserved and internal features are imaged clearly. 